\title{
SARS Coronavirus Papain-Like Protease Inhibits the TLR7 Signaling Pathway through Removing Lys63-Linked Polyubiquitination of TRAF3 and TRAF6
}

\author{
Shih-Wen Li ${ }^{1}$, Ching-Ying Wang ${ }^{1}$, Yu-Jen Jou ${ }^{1}$, Su-Hua Huang ${ }^{2}$, Li-Hsin Hsiao ${ }^{1}$, Lei Wan ${ }^{3}$, \\ Ying-Ju Lin ${ }^{3}$, Szu-Hao Kung ${ }^{4}$ and Cheng-Wen Lin ${ }^{1,2, *}$ \\ 1 Department of Medical Laboratory Science and Biotechnology, China Medical University, Taichung 404, \\ Taiwan; violet7053@gmail.com (S.-W.L.); spirit1126@hotmail.com (C.-Y.W.); alvajou@gmail.com (Y.-J.J.); \\ bonny6789@gmail.com (L.-H.H.) \\ 2 Department of Biotechnology, College of Health Science, Asia University, Wufeng, Taichung 413, Taiwan; \\ shhuang@asia.edu.tw \\ 3 Department of Medical Genetics and Medical Research, China Medical University Hospital, Taichung 404, \\ Taiwan; lei.joseph@gmail.com (L.W.); yjlin.kath@gmail.com (Y.-J.L.) \\ 4 Department of Biotechnology and Laboratory Science in Medicine, National Yang Ming University, \\ Taipei 112, Taiwan; szkung@ym.edu.tw \\ * Correspondence: cwlin@mail.cmu.edu.tw; Tel.: +886-4-2205-3366 (ext. 7210); Fax: +886-4-2205-7414
}

Academic Editor: Atsushi Matsuzawa

Received: 14 March 2016; Accepted: 26 April 2016; Published: 5 May 2016

\begin{abstract}
Severe acute respiratory syndrome coronavirus (SARS-CoV) papain-like protease (PLPro) reportedly inhibits the production of type I interferons (IFNs) and pro-inflammatory cytokines in Toll-like receptor 3 (TLR3) and retinoic acid-inducible gene 1 (RIG-I) pathways. The study investigated the inhibitory effect and its antagonistic mechanism of SARS-CoV PLPro on TLR7-mediated cytokine production. TLR7 agonist (imiquimod (IMQ)) concentration-dependently induced activation of ISRE-, NF- $\kappa$ B- and AP-1-luciferase reporters, as well as the production of IFN- $\alpha$, IFN- $\beta$, TNF- $\alpha$, IL- 6 and IL-8 in human promonocyte cells. However, SARS-CoV PLPro significantly inhibited IMQ-induced cytokine production through suppressing the activation of transcription factors IRF-3, NF- $\kappa \mathrm{B}$ and AP-1. Western blot analysis with anti-Lys48 and anti-Lys63 ubiquitin antibodies indicated the SARS-CoV PLPro removed Lys63-linked ubiquitin chains of TRAF3 and TRAF6, but not Lys48-linked ubiquitin chains in un-treated and treated cells. The decrease in the activated state of TRAF3 and TRAF6 correlated with the inactivation of TBK1 in response to IMQ by PLPro. The results revealed that the antagonism of SARS-CoV PLPro on TLR7-mediated innate immunity was associated with the negative regulation of TRAF3/6-TBK1-IRF3/NF-kB/AP1 signals.
\end{abstract}

Keywords: SARS-CoV; Toll-like receptor 7; imiquimod; TRAF3; TRAF6; Lys63-linked polyubiquitin chains

\section{Introduction}

Toll-like receptor 7 (TLR7) is one of the well-known pattern recognition receptors [1], sensing single-stranded RNA viruses via the recognition of the viral RNA genome [2]. Binding of the ligand to the TLR extracellular domain causes the homodimerization of TLRs via further interaction of the intracellular Toll/interleukin-1 receptor (TIR) domain, then activates myeloid differentiation factor 88 (MyD88)-dependent and/or TIR domain-containing adaptor-inducing IFN- $\beta$ (TRIF)-dependent pathways [1,3]. In the MyD88-dependent pathway, the complex of MyD88 with activated TLR dimers, except for TLR3, recruits IL-1 receptor-associated kinase (IRAK)-4, then leads IRAK4 to activate other members of the IRAK, like IRAK1 and IRAK2. The activated IRAKs directly interact with TNF 
receptor-associated factor (TRAF) 6 (E3 ubiquitin ligase) plus E2 ubiquitin-conjugating enzymes Ubc13 and Uev1A, resulting in Lys63-linked ubiquitination of TRAF6, IRAKs and TGF- $\beta$-activated kinase 1 (TAK1) [4]. In the TRIF-dependent pathway, activated TLR3 directly binds TRIF, resulting in the activation of TRIF connecting with TRAF3 to turn on TANK-binding kinase 1 (TBK1) and IKKs for IRF3/7 phosphorylation [4,5]. Alternatively, TRIF associates with RIP1 and then forms the complex along with TRAF6 for activation of TAK1. Ubiquitin-activated TAK1 phosphorylates mitogen-activated protein kinases (MAPKs) and IкB-kinases (IKKs), initiating AP-1, NF- $\kappa B$ and IRF3/7 signaling on the production of cytokines, chemokines and type I interferons (IFNs).

Severe acute respiratory syndrome (SARS)-associated coronavirus (SARS-CoV) causes the pro-inflammatory cytokine storms, recruitment of immune responder cells into the lungs, acute respiratory distress syndrome (ARDS) and even lung fibrosis in the late phase [6,7]. Among 14 ORFs encoded by SARS-CoV [8,9], ORF1a/ORF1ab is the biggest one encoding polyprotein replicases 1a and $1 \mathrm{ab}$, primarily involved in SARS-CoV replication. Specifically, ORF1a-encoded papain-like protease (PLPro) and 3C-like protease (3CLpro) process cis- and trans-cleavage activities on replicases 1a and $1 \mathrm{ab}$ for creating 16 nonstructural (NS) proteins (termed NS 1 through NS16). PLPro, recognizing a consensus motif LXGG as a de-ubiquitinating/de-ISGylating enzyme [10-13], exhibits the antagonistic activities of type I interferon (IFN). PLPro directly interacts with IFN regulatory factor 3 (IRF-3) to block IRF-3 phosphorylation and nuclear translocation [14,15]. Conversely, another study indicated that type I IFN antagonism of PLPro is not correlated to a direct interaction of PLPro with IRF-3 or affecting the phosphorylation of IRF3, but PLPro suppresses the NF- $\mathrm{KB}$ signaling pathway by preventing I $\mathrm{K} B \alpha$ degradation [15]. Recent studies demonstrate that PLPro disrupts the stimulator of interferon gene (STING)-mediated signaling and then negatively regulates type I IFN induction [16,17]. PLPro physically interacts with the STING-TRAF3-TBK1 complex, reducing the ubiquitinated forms of STING, RIG-I, TRAF3, TBK1 and IRF-3. Similarly, de-ubiquitinating enzyme cylindromatosis (CYLD) inhibits NF-KB signaling via de-ubiquitination and inactivation of TNFR-associated factor 2 (TRAF2) and TRAF6 [18,19]; de-ubiquitinating protease A20 inhibits NF- $\mathrm{KB}$ activation induced by Toll-like receptor 4 (TLR4) via removing K63-linked polyubiquitin chains of TRAF6 [20]. PLPro interacts with above key regulators of TLR signal pathways; thus, characterizing the antagonistic mechanisms of TLR signal pathways by SARS-CoV PLPro could provide valuable insights into SARS pathogenesis.

SARS-CoV-specific GU-rich ssRNA has been demonstrated to be recognized by TLR7, establishing a link with the induction of pro-inflammatory cytokines TNF- $\alpha$, IL-6 and IL-12 [21]. SARS-CoV infection rapidly activates TLR7 signaling in plasmacytoid dendritic cells; however, some SARS-CoV proteins subsequently inhibit and/or modulate type I IFN responses in plasmacytoid dendritic cells [22]. Therefore, the effect of the unique SARS-CoV proteins on the activation of the TLR7 signaling pathway is noteworthy to elucidate.

This study assesses possible effects of PLPro on TLR7 signaling pathways and the production of type I IFNs and pro-inflammatory cytokines. In this study, PLPro-expressing and vector control cells were treated with TLR7 agonist (imiquimod (IMQ)) and then examined on IRF3, STAT1, NF-kB, p38 MAPK and c-Jun regulation using dual luciferase reporters, Western blotting and real-time PCR assays. Analysis of ubiquitin-modified proteins reveals that the change in ubiquitination of TRAF3 and TRFA6 played the crucial role in the antagonistic mechanisms of TLR7-mediated type I IFN induction and NF- $k B$ activation by PLPro.

\section{Results}

\subsection{PLPro Suppressed TLR7 Agonist-Induced Production of Type I IFNs in Human Promonocytes}

To examine whether SARS-CoV PLPro modulates the TLR7 signaling pathway, stable transfected promonocyte cells expressing PLPro and vector control cells were established, treated with TLR7 agonist (imiquimod (IMQ)), then further analyzed for activation of type I IFN production (Figure 1). Western blot analysis showed a $60-\mathrm{kDa}$ band recombinant PLPro protein in transfected cells, but not 
controls, as well as the similar expression level of TLR7 in both types of cells (Figure 1A), demonstrating that SARS-CoV PLPro was stably expressed in human promonocyte cells that did not alter the TLR7 expression. Quantitative real-time PCR signified TLR7 agonist treatment stimulating higher transcriptional levels of IFN- $\alpha$ and IFN- $\beta$ in vector controls than in PLPro-expressing cells (Figure 1B,C). Western blotting analysis of both of these cells indicated that IRF3 phosphorylation in vector control cells reached a maximum level at $30 \mathrm{~min}$, but disappeared at $60 \mathrm{~min}$ after IMQ treatment. Importantly, PLPro inhibited IMQ-induced activation of IRF3 phosphorylation since no significant change in IRF3 phosphorylation was detectable at $30 \mathrm{~min}$ after IMQ treatment compared to non-treatment (Figure 1D). To assess the functional activity of IFN- $\alpha$ and IFN- $\beta$ induced by TLR7 agonist, the interferon-stimulated response element (ISRE) reporter of the dual-luciferase assay was further performed (Figure 2A). The TLR7 agonist dose-dependently triggered the ISRE promoter activity in vector control cells, but not in PLPro-expressing cells (Figure 2A). Quantitative real-time PCR signified that the TLR7 agonist dose-dependently upregulated the IFN-stimulated genes (ISGs), including PKR and IRF7, in vector controls, but not PLPro-expressing cells (Figure 2B,C). In addition, TLR7 agonist-induced activation of STAT1 was delayed and suppressed by SARS-CoV PLPro (Figure 2D). Results revealed that PLPro suppresses TLR7 agonist-induced production of type I IFNs through inhibiting the IRF-3 activation in the TLR7 signaling pathway.

A.

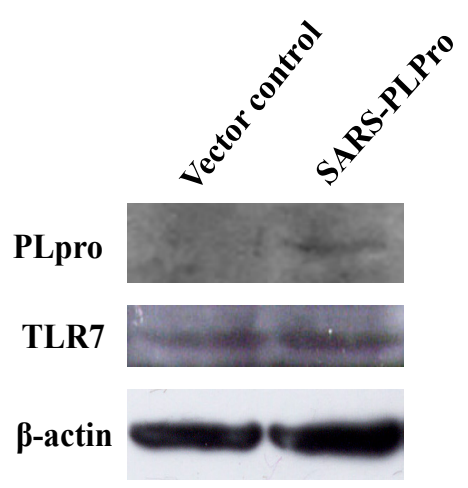

C.

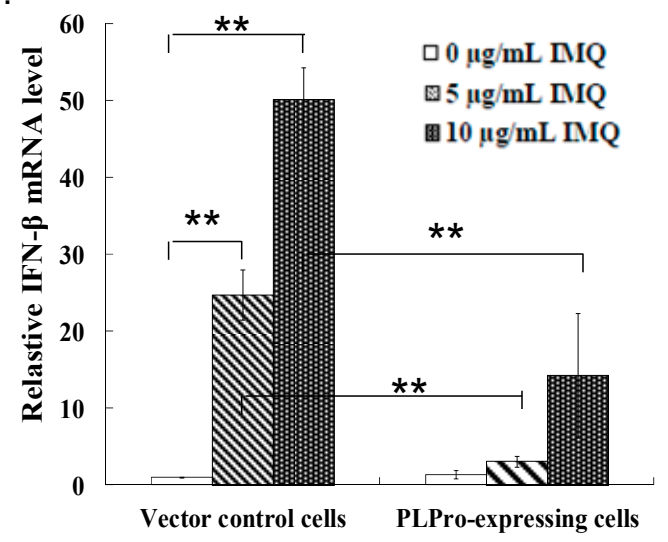

B.

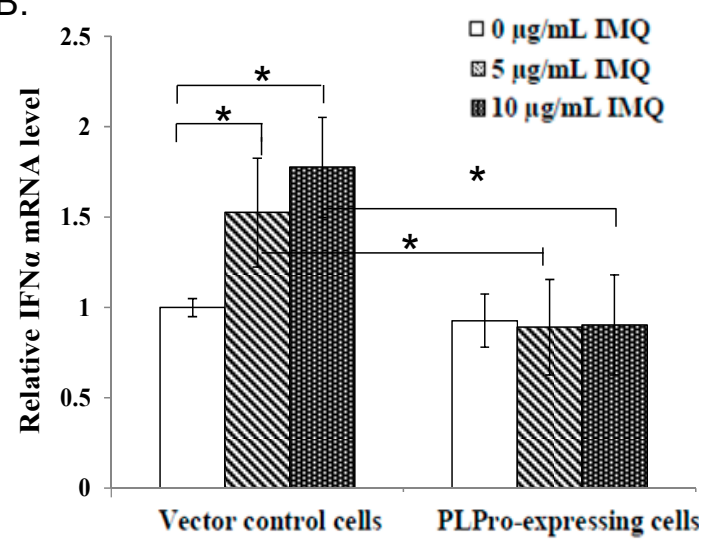

D.

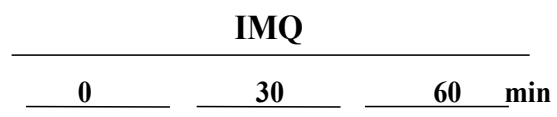

Vector PLPro Vector PLPro Vector PLPro

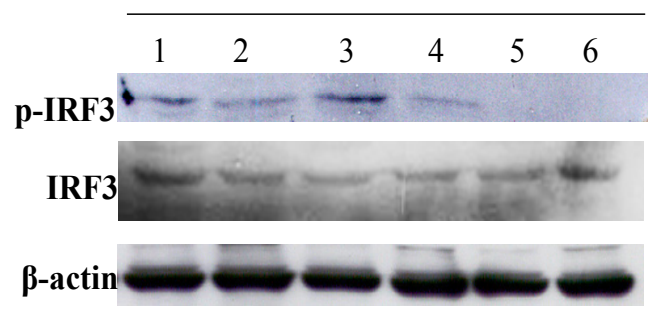

Figure 1. Effect of SARS-CoV PLPro on TLR7 agonist-induced production of type I IFNs via IRF3 signaling. The expression level of PLPro and TLR7 in the vector control and PLPro-expressing cells was analyzed using Western blot assay (A). Both types of transfected cells were treated with or without imiquimod (IMQ) for $4 \mathrm{~h}$, and then, their mRNA levels of IFN- $\alpha$ and IFN- $\beta$ were measured by quantitative PCR. Relative mRNA levels of IFN- $\alpha$ (B) and IFN- $\beta$ (C) were normalized by GAPDH mRNA, presented as a relative ratio. To determine IRF3 activation, the lysates were also analyzed using Western blot with anti-phospho-IRF3 antibodies (D). ${ }^{*} p$-Value $<0.05$; ${ }^{* *} p$-value $<0.01$ by Student's $t$-test. 
A.

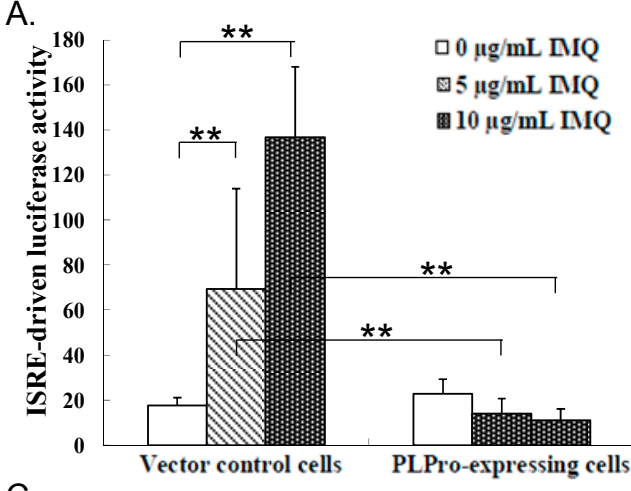

C.

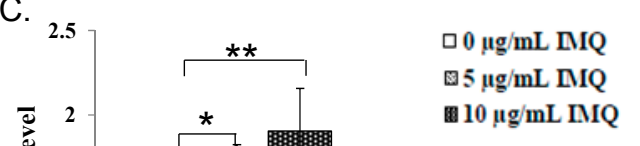

B.

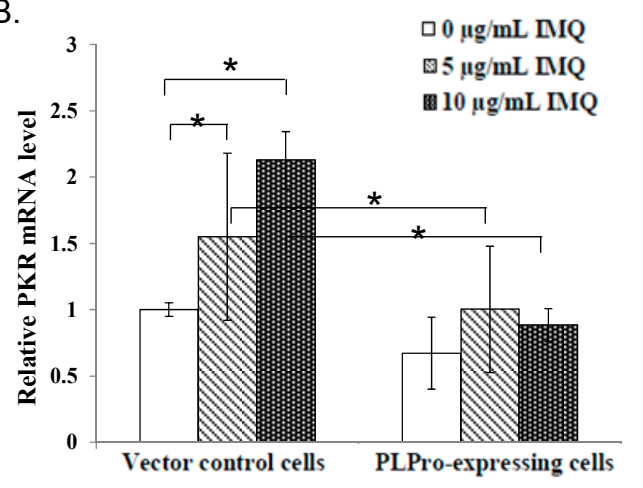

D.

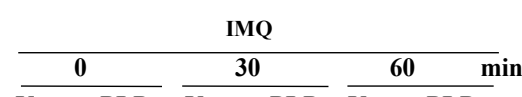

$\overline{\text { Vector PLPro }} \frac{30}{\text { Vector PLPro Vector PLPro }}$

$\begin{array}{llllll}1 & 2 & 3 & 4 & 5 & 6\end{array}$

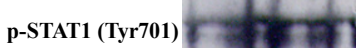

STAT1

$\beta$-actin

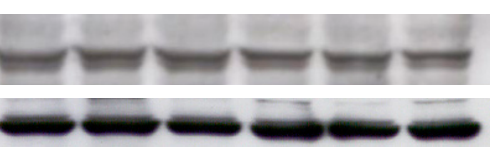

Figure 2. Inhibitory effect of SARS-CoV PLPro on TLR7 agonist-induced activation of type I IFN signaling. ISRE-driven luciferase reporter activity and the mRNA levels of PKR and IRF7 were determined $4 \mathrm{~h}$ post-IMQ treatment. ISRE-driven firefly luciferase activity was normalized by Renilla luciferase activity (A). Relative mRNA levels of PKR (B) and IRF7 (C) were normalized by GAPDH mRNA, presented as a relative ratio. In addition, the activated status of STAT1 was examined using Western blot with anti-phospho-STAT1 (Tyr701) antibodies (D). ${ }^{*} p$-Value $<0.05$; ${ }^{* *} p$-value $<0.01$ by Student's $t$-test.

\subsection{Inhibition of TLR7 Agonist-Induced Pro-Inflammatory Cytokines by SARS-CoV PLPro}

Besides type I IFN production, we checked whether the TLR7 agonist upregulates pro-inflammatory cytokines. The effect of SARS-CoV PLPro on the activation of NF-KB and AP1 promoters, as well as the production of pro-inflammatory cytokines (IL-6, IL-8 and TNF- $\alpha$ ) was further characterized via dual-luciferase reporter assay and SYBR green real-time PCR (Figures 3 and 4). Results indicated that SARS-CoV PLPro inhibits TLR7 agonist-induced activation of NF-kB and AP1 promoters, which was associated with reducing the stimulated upregulation of IL-6, IL-8 and TNF- $\alpha$ mRNA (Figures $3 \mathrm{~A}, \mathrm{~B}$ and $4 \mathrm{~A}-\mathrm{C}$ ). To explore the inhibitory effect of PLPro on NF- $\mathrm{kB}$ and AP1 signals, the levels of NF- $\mathrm{kB}$ p65, p38 MAPK and c-Jun phosphorylation were subsequently detected in responses to TLR7 agonist using Western blotting (Figures $3 C$ and $4 \mathrm{D}$, respectively). Western blotting demonstrated that PLPro suppressed TLR7 agonist-induced phosphorylation of NF- $\mathrm{kB}$, p38 MAPK and c-Jun compared to those in the vector control at $30 \mathrm{~min}$ post-treatment. The results demonstrated that SARS-CoV PLPro suppressed TLR7-induced cytokines through decreasing the phospho-NF-kB p65, p38 MAPK and c-Jun, resulting in suppressing of the NF- $\mathrm{kB}$ and AP-1 signaling pathway. 

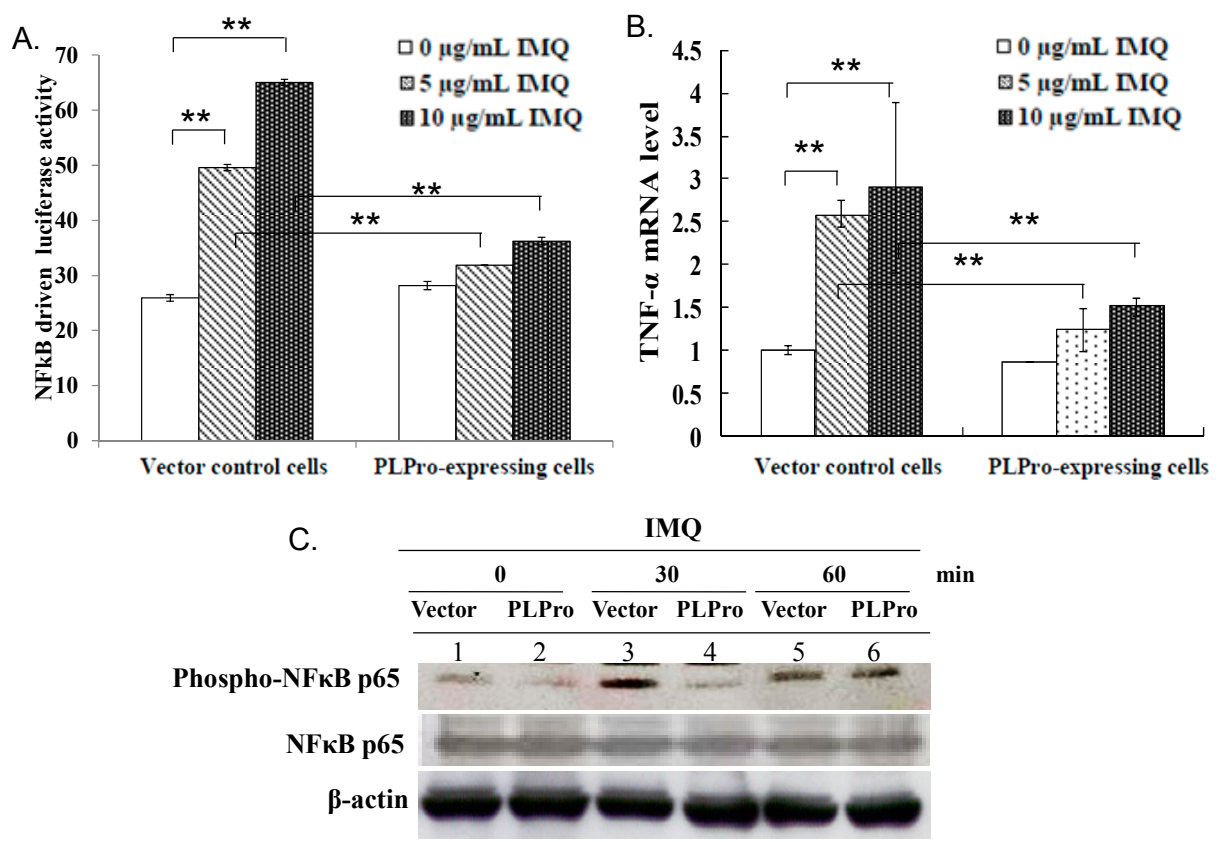

Figure 3. Inhibition of IMQ-induced TNF- $\alpha$ production via NF- $\kappa B$ signaling by SARS-CoV PLPro. Both types of cells were treated with(out) IMQ for $4 \mathrm{~h}$, and then, NF-kB-driven luciferase reporter activity and the TNF- $\alpha$ mRNA level were determined using the dual luciferase reporter assay (A) and quantitative PCR (B), respectively. For determining NF- $\kappa$ B activation, the lysates were also analyzed using Western blot with anti-phospho-NF-kB p65 antibodies (C). ${ }^{* *} p$-Value $<0.01$ by Student's $t$-test.
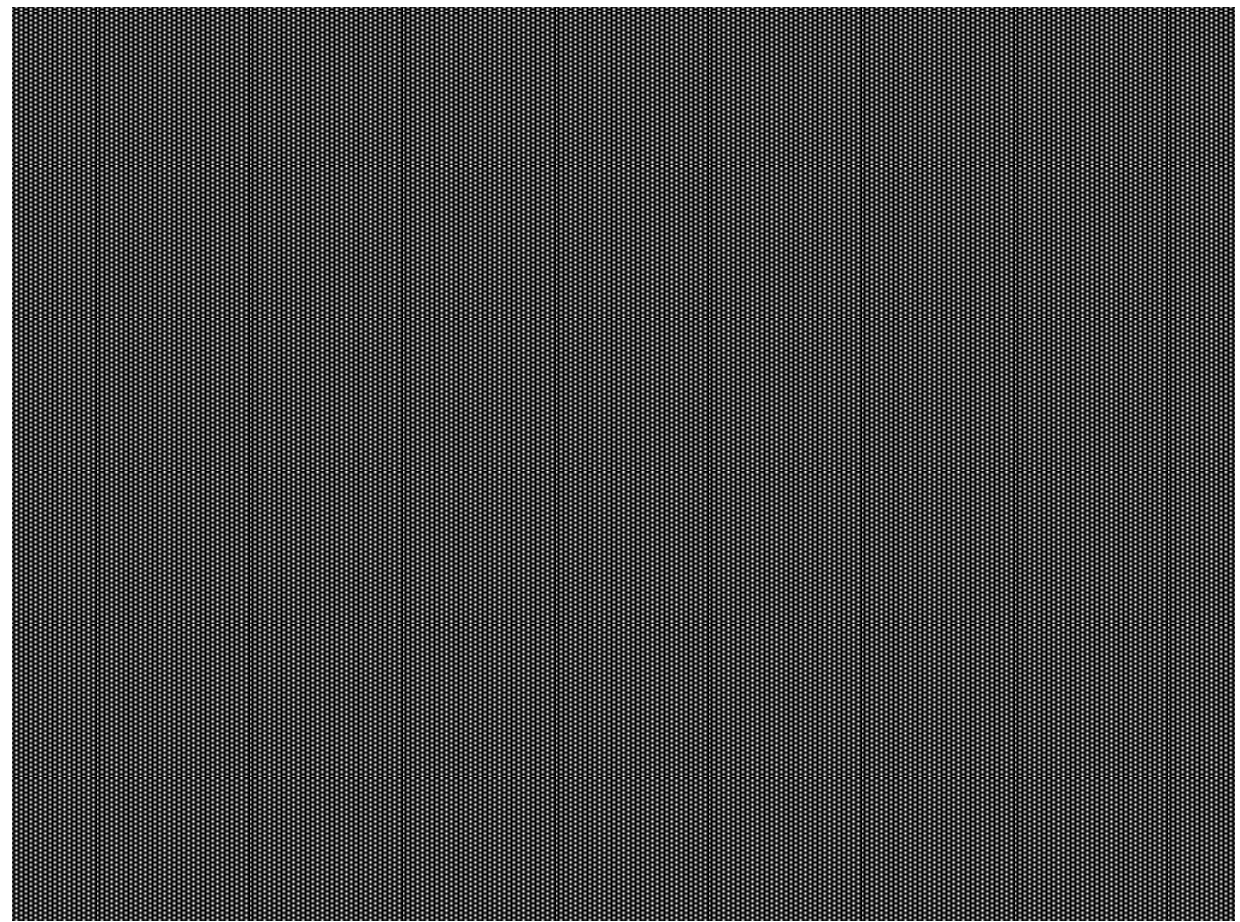

D.

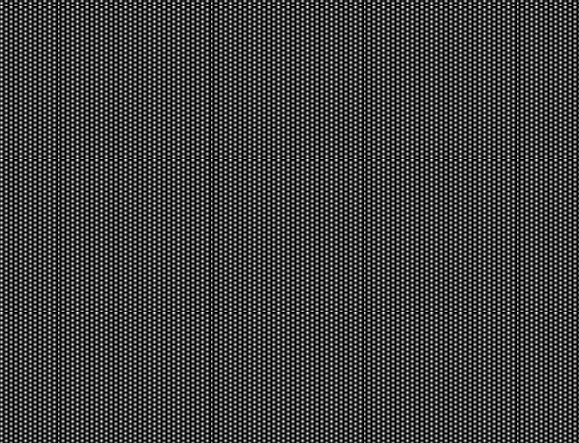

Figure 4. Detection of IMQ-induced AP-1-mediated production of IL-6 and IL-8 in the vector control and PLPro-expressing cells. AP-1-driven firefly luciferase activity was normalized by Renilla luciferase activity (A). Relative mRNA levels of IL-6 (B) and IL-8 (C) were normalized by GAPDH mRNA, presented as a relative ratio. In addition, the activated status of p38 MAPK and AP-1 was examined using Western blot with anti-phospho-p38 MAPK and anti-phospho-c-Jun antibodies (D). * $p$-Value $<0.05 ;{ }^{* *} p$-value $<0.01$ by Student's $t$-test. 


\subsection{Ubiquitin Removal of TRAF3 and TRAF6 Responsible for the TLR7 Antagonism of SARS-CoV PLPro}

To examine the inhibitory mechanism of TLR7 antagonism by SARS-CoV PLPro, differential profiles of ubiquitin-conjugated proteins in the vector control and PLPro-expressing cells in the absence or presence of IMQ were analyzed using immune-precipitation (Figure 5A,B). Because Lys63-linked auto-ubiquitination of TRAF is required for its E3 ubiquitin ligase activity and SARS-CoV PLPro proceeds the de-ubiquitination enzymatic activity, the ubiquitination status of TRAF3 and TRAF6 was subsequently analyzed for whether they were involved in the TLR7 signaling pathway induced by IMQ (Figure 5). The immunoprecipitation assay indicated that PLPro reduced Lys48- and Lys63-linked poly-ubiquitination of TRAF3 and TRAF6 induced by IMQ (Figure 5A,B, Lane 2 vs. 4). Subsequently, Western blotting analysis indicated that IMQ-induced activation of TBK1 was suppressed in PLPro-expressing cells (Figure 5C, Lane 3 vs. Lane 4; Figure 5D). Therefore, the results indicated that PLPro reduced Lys63-linked poly-ubiquitination of TRAF3 and TRAF6, which correlated with the activation of TBK1, p38 MAPK, NF-KB and IRF3 in the TLR7 signaling pathway.

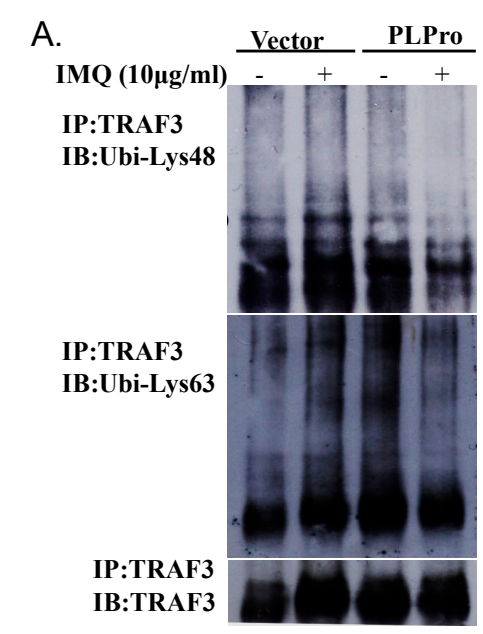

C.

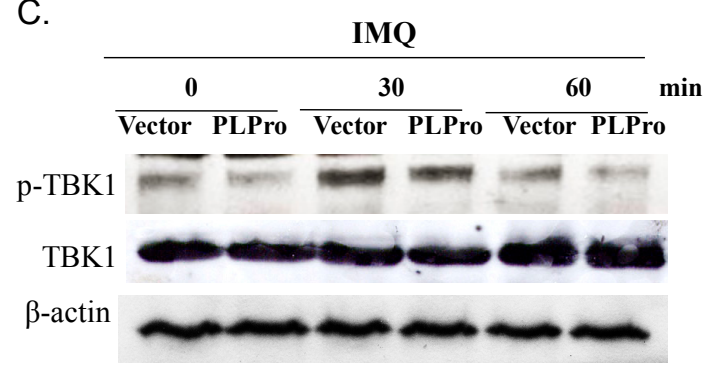

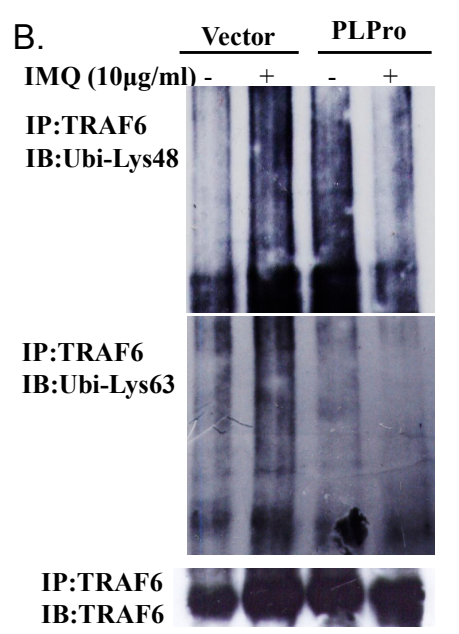

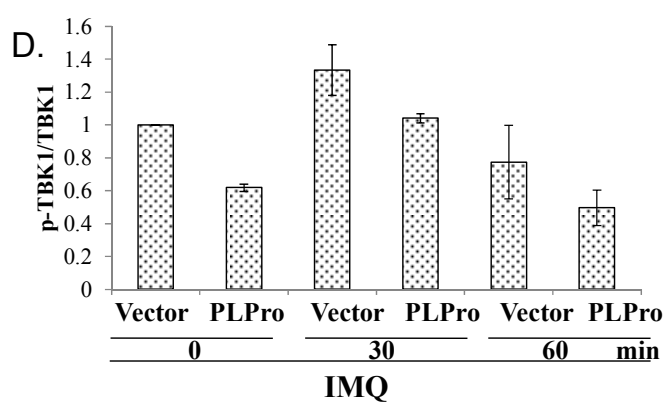

Figure 5. Detecting the Lys48- and Lys63-linked ubiquitination of TRAF3 and TRAF6 measured by the immunoprecipitation assay. Vector control and PLPro-expressing cells were treated with(out) IMQ for 1 day; cell lysates were immunoprecipitated with anti-TRAF3 (A) or anti-TRAF6 (B) followed by Western blotting probed with either anti-Lys48 ubiquitin or anti-Lys63 ubiquitin antibodies. Phospho-TBK1 levels were detected by Western blot (C). The relative band intensity of phospho-TBK1 was normalized by TBK1, compared to the mock vector control cells, and quantified using ImageJ based on triplicate replicates of each experiment (D).

\section{Discussion}

This study was the first report in which SARS-CoV PLPro showed antagonistic activity against TLR7 agonist-induced production of IFN- $\alpha$, IFN- $\beta$, TNF- $\alpha$, IL- 6 and IL- 8 via reducing TLR7 agonist-induced phosphorylation of transcription factors IRF3, NF- $k B$ and c-Jun (Figures 1, 3 and 4). TLR7 agonist-induced IFN- $\beta$ stimulated the transcriptional activity of STAT1 to upregulate the 
expression of ISGs, such as PKR and IRF7, in vector control cells (Figure 2). However, there was no significant response of STAT1-mediated signaling due to the inhibitory effect of PLPro on the production of TLR7 agonist-induced IFN- $\beta$ (Figure 2). The results were constant with previous reports in that SARS-CoV PLPro inhibited TLR3- and RIG-I-mediated production of type I interferons and proinflammatory cytokines via inactivating the transcription factors IRF3 and NF- $\mathrm{KB}[15,23]$.

This study proposed TRAF3 and TRAF6 as the crucial regulators of PLPro antagonism via modification of the profiles of ubiquitin-conjugate proteins in the vector control and PLPro-expressing cells; thus, the ubiquitin linkage status of TRAF3 and TRAF6 was analyzed post-treatment with(out) IMQ using immunoprecipitation (Figure 5). Immunoprecipitation assays indicated that PLPro decreased the Lys63-linked polyubiquitin chains of TRAF3 and TRAF6, causing the decrease in the E3 ubiquitin ligase activity of TRAF3 and TRAF6, which correlated with lower levels of TBK-1 phosphorylation in PLPro-expressing cells than vector control cells in response to IMQ. PLPro has been reported to upregulate the ubiquitin-conjugating enzyme E2-25k and proteasome subunit alpha type 5, which resulted in the increase of ERK1 poly-ubiquitination in PLPro-expressing cells as the type I IFN antagonistic mechanism of SARS PLPro via the degradation of ERK1 [24,25]. The high ubiquitination level of TRAF3 and TRAF6 in PLPro-expressing cells (Figure 5A,B) might be responsible for the activation of the ubiquitin proteasome pathway, as demonstrated in our prior reports $[24,25]$. The finding was also similar to previous reports in that the inhibitory effect of SARS-CoV PLPro on the inactivation of the transcription factors IRF3 and NF- $\mathrm{KB}$ has been demonstrated to correlate with PLPro-reduced ubiquitination of RIG-I, STING TRAF3, TBK1 and IRF3 in the TLR3 and RIG-I pathways [16,17]. These previous reports proposed an antagonistic mechanism of SARS-CoV PLPro, including the inhibition of STING-MAVS-TBK1/IKK $\varepsilon$ and STING-TRAF3-TBK1 signaling in the TLR3 and RIG-I pathways. Since TBK1 has been suggested to activate protein phosphatases [26], no detectable level of IRF3 phosphorylation, as well as the decrease of NF- $\mathrm{kB}$ p65, p38 MAPK and c-Jun phosphorylation in the vector control and PLPro-expressing cells at $60 \mathrm{~min}$ post-IMQ treatment (Figures 1D and 3C) could be due to a the negative feedback of the TBK1-IRF3/NF- $\mathrm{KB}$ pathways. Therefore, we propose that the inhibition of TRAF3/6-TBK1-IRF3/NF-KB/AP1 signals by PLPro is responsible for the antagonistic mechanism of SARS-CoV PLPro against TLR7-medaited production of type I IFNs and pro-inflammatory cytokines.

\section{Materials and Methods}

\subsection{Cell Line and Western Blot Assay}

SARS-CoV PLPro-expressing and empty vector control promonocyte cell lines were established, as described in our previous reports [24,25]. Both stably-transfected cell lines grew in RPMI-1640 medium containing 10\% FBS and $800 \mu \mathrm{g} / \mathrm{mL}$ of Geneticin ${ }^{\circledR}$ Selective Antibiotic (G418 Sulfate). The expression level of PLPro and TLR7 in both stable cell lines was analyzed using Western Blotting with anti-PLPro mouse sera and anti-TLR7 mAb. To monitor the activation of the IMQ-induced TLR7 signaling pathway, PLPro-expressing and vector control cells were treated with $10 \mu \mathrm{M}$ IMQ (InvivoGen, San Diego, CA, USA) and harvested 0, 30 and 60 min post-treatment. Lysate from IMQ-treated cells was used for the Western blotting assay; the resulting blots were probed with specific primary antibodies against IRF3, phosphor-IRF3, STAT1, phospho-STAT1(Tyr701), NF-kB p65, phospho-NF-kB p65, p38 MAPK, phospho-p38 MAPK, c-Jun, phospho-c-Jun, TBK1, phospho-TBK1, TRAF3, TRAF6 and anti- $\beta$-actin $\mathrm{mAb}$ (Abcam, Cambridge, UK). Immune complexes were detected with horseradish peroxidase-conjugated secondary antibodies, followed by enhanced chemiluminescence detection (Amersham Pharmacia Biotech, Piscataway, NJ, USA).

\subsection{Signaling Pathway Assays with the Dual Luciferase Reporter System}

Cis-reporter plasmids pISRE-Luc, pAP-1-Luc and pNF-kB-Luc were used to examine the firefly luciferase activity driven from each cis-acting transcriptional element. SARS-CoV PLPro-expressing 
and vector control cells were transfected with the indicated cis-reporter plasmid, plus internal control reporter pRluc-C1 (BioSignal Packard, Montréal, QC, Canada) using GenePorter reagent. After $4 \mathrm{~h}$ of incubation with or without IMQ, the activity of experimental firefly luciferase and control Renilla luciferase was measured by the dual Luciferase Reporter Assay System (Promega, Madison, WI, USA) and the Clarity ${ }^{\mathrm{TM}}$ Luminescence Microplate Reader (BioTek Instruments, Bad Friedrichshall, Germany) [27].

\subsection{Quantitative Expression Analysis of Type I IFNs and Pro-Inflammatory Cytokines Using Real-Time PCR}

PLPro-expressing or empty vector cells were incubated for $4 \mathrm{~h}$ in the presence or absence of IMQ and harvested for isolation of total RNAs by a PureLink Micro-to-Midi Total RNA Purification System Kit (Invitrogen, Carlsbad, CA, USA), following quantification of gene expression by a two-step real-time PCR using SYBR Green I. First, cDNA was synthesized from $1 \mu \mathrm{g}$ total RNA, using oligonucleotide $\mathrm{d}(\mathrm{T})$ primer and a SuperScript III reverse transcriptase kit (Invitrogen). Each real-time PCR reaction contained $5 \mu \mathrm{L}$ of a cDNA mixture, $1 \mu \mathrm{L}$ of primer pair $(200 \mathrm{nM})$ and 12.5 $\mu \mathrm{L}$ Smart Quant Green Master Mix with Dutp and ROX (Protech, Vancouver, WA, USA). Primer pairs included (1) 5'-CAACCAGCGGTTGACTTTTT-3' and 5'-ATCCAGGAAGGCAAACTGAA-3' for PKR; (2) $5^{\prime}$-GTGAGGAAATACTTCCAAAGAATCAC- $3^{\prime}$ and $5^{\prime}$-TCTCATGATTTCTGCTCTGACAA- ${ }^{\prime}$ for IFN- $\alpha$; (3) $5^{\prime}$-AACTGCAACCTTTCGAAGCC- $3^{\prime}$ and $5^{\prime}$-TGTCGCCTACTACCTGTTGTGC- $3^{\prime}$ for IFN- $\beta$; (4) $5^{\prime}$-CGCGGCACTAACGACAGGCGAG- $3^{\prime}$ and $5^{\prime}$-GCTGCCGTGCCCGGAATTCCAC- $3^{\prime}$ for IRF7; (5) $5^{\prime}$-CTTCTCCTTCCTGATCGTGG-3 $3^{\prime}$ and $5^{\prime}$-GCTGGTTATCTCTCAGCTCCA- $3^{\prime}$ for TNF- $\alpha$; (6) $5^{\prime}$-GATGGATGCTTCCAATCTGGAT- ${ }^{\prime}$ and $5^{\prime}$-AGTTCTCCATAGAGAACAACATA- $3^{\prime}$ for IL-6; (7) 5'-CGATGTCAGTGCATAAAGACA-3 ${ }^{\prime}$ and $5^{\prime}$-TGAATTCTCAGCCCTCTTCAAAAA-3' for IL-8; and (8) 5' -AGCCACATCGCTCAGACAC-3' and 5'-GCCCAATACGACCAAATCC-3' for glyceraldehyde-3-phosphate dehydrogenase (GAPDH). Real-time PCR was performed as described in our previous reports $[23,24]$. The amplification and detection of specific products were conducted in an ABI Prism 7900 sequence detection system (PE Applied Biosystems, Carlsbad, CA, USA). Relative changes in the mRNA level of indicated genes were normalized relative to GAPDH mRNA.

\subsection{Immunoprecipitation Assays}

To detect ubiquitination of TRAF3 and TRAF6, lysates from PLPro-expressing or vector cells with or without IMQ were collected, incubated with specific monoclonal antibodies anti-TRAF3 or anti-TRAF6 for $4 \mathrm{~h}$ at $4{ }^{\circ} \mathrm{C}$ and then followed by the addition of protein A-Sepharose beads and an additional $2 \mathrm{~h}$ of incubation. After centrifugation, pellets were washed four times with NET buffer ( $150 \mathrm{mM} \mathrm{NaCl}, 0.1 \mathrm{mM}$ EDTA, $30 \mathrm{mM}$ Tris- $\mathrm{HCl}$, pH 7.4); immunoprecipitated proteins were dissolved in $2 \times$ SDS-PAGE sample buffers without 2-mercaptoethanol, boiled for $10 \mathrm{~min}$, separated by SDS-PAGE and then transferred to nitrocellulose. Resulting blots were blocked with 5\% skim milk, reacted with specific monoclonal antibodies anti-TRAF3, anti-TRAF6, anti-Lys48- or anti-Lys63-linked ubiquitin and then followed by enhanced chemiluminescence detection.

\subsection{Statistical Analysis}

Data were from three independent experiments; error bars represent the standard error of the mean. Chi-square and Student's $t$-test were used to analyze all data, with statistical significance between types of cells noted at $p<0.05$.

\section{Conclusions}

In summary, SARS-CoV PLPro inhibits TLR7-mediated signaling, leading to reducing of the cytokine production during antiviral responses. PLPro diminishes Lys63-linked ubiquitination of TRAF3 and TRAF6 and then inactivates their downstream molecules, such as kinases (TBK1 and p38 MAPK) and transcription factors (IRF3, NF- $\mathrm{KB}$ and AP-1). The results let us conclude that SARS-CoV 
PLPro negatively regulates the TRAF3/6-TBK1-IRF3/NF- KB/AP1 signals in TLR7-mediated antiviral and inflammatory responses.

Acknowledgments: This work was supported by the Ministry of Science and Technology, Taiwan (MOST 101-2320-B-039-036-MY3, MOST 102-2628-B-039-044-MY3) and China Medical University (CMU101-ASIA-05, CMU102-ASIA-15 and CMU103-ASIA-07).

Author Contributions: Cheng-Wen Lin conceived the project, contributed to experimental designs, performed experiments, interpreted the results, generated figures and wrote the manuscript; Shih-Wen Li, Ching-Ying Wang, Yu-Jen Jou, and Li-Hsin Hsiao performed the experiments, interpreted the results, and generated figures. Su-Hua Huang, Lei Wan, Ying-Ju Lin, and Szu-Hao Kung interpreted the results and wrote the manuscript. All authors approved the manuscript.

Conflicts of Interest: The authors declare no conflict of interest.

\section{References}

1. Brown, J.; Wang, H.; Hajishengallis, G.N.; Martin, M. LR-signaling networks: An integration of adaptor molecules, kinases, and cross-talk. J. Dent. Res. 2011, 90, 417-427. [CrossRef] [PubMed]

2. Kumar, H.; Kawai, T.; Akira, S. Toll-like receptors and innate immunity. Biochem. Biophys. Res. Commun. 2009, 388, 621-625. [CrossRef] [PubMed]

3. Yang, L.; Seki, E. Toll-like receptors in liver fibrosis: Cellular crosstalk and mechanisms. Front. Physiol. 2012, 3, 138. [CrossRef] [PubMed]

4. Takeuchi, O.; Akira, S. Pattern recognition receptors and inflammation. Cell 2010, 140, 805-820. [CrossRef] [PubMed]

5. Yamamoto, M.; Takeda, K. Current views of toll-like receptor signaling pathways. Gastroenterol. Res. Pract. 2010, 2010, 240365. [CrossRef] [PubMed]

6. Huang, K.J.; Su, I.J.; Theron, M.; Wu, Y.C.; Lai, S.K.; Liu, C.C.; Lei, H.Y. An interferon- $\gamma$-related cytokine storm in SARS patients. J. Med. Virol. 2005, 75, 185-194. [CrossRef] [PubMed]

7. He, L.; Ding, Y.; Zhang, Q.; Che, X.; He, Y.; Shen, H.; Wang, H.; Li, Z.; Zhao, L.; Geng, J.; et al. Expression of elevated levels of pro-inflammatory cytokines in SARS-CoV-infected ACE2 ${ }^{+}$cells in SARS patients: Relation to the acute lung injury and pathogenesis of SARS. J. Pathol. 2006, 210, 288-297. [CrossRef] [PubMed]

8. Rota, P.A.; Oberste, M.S.; Monroe, S.S.; Nix, W.A.; Campagnoli, R.; Icenogle, J.P.; Peñaranda, S.; Bankamp, B.; Maher, K.; Chen, M.H.; et al. Characterization of a novel coronavirus associated with severe acute respiratory syndrome. Science 2003, 300, 1394-1399. [CrossRef] [PubMed]

9. Ziebuhr, J. Molecular biology of severe acute respiratory syndrome coronavirus. Curr. Opin. Microbiol. 2004, 7, 412-419. [CrossRef] [PubMed]

10. Barretto, N.; Jukneliene, D.; Ratia, K.; Chen, Z.; Mesecar, A.D.; Baker, S.C. The papain-like protease of severe acute respiratory syndrome coronavirus has deubiquitinating activity. J. Virol. 2005, 79, 15189-15198. [CrossRef] [PubMed]

11. Lindner, H.A.; Fotouhi-Ardakani, N.; Lytvyn, V.; Lachance, P.; Sulea, T.; Ménard, R. The papain-like protease from the severe acute respiratory syndrome coronavirus is a deubiquitinating enzyme. J. Virol. 2005, 79, 15199-15208. [CrossRef] [PubMed]

12. Sulea, T.; Lindner, H.A.; Purisima, E.O.; Menard, R. Deubiquitination, a new function of the severe acute respiratory syndrome coronavirus papain-like protease? J. Virol. 2005, 79, 4550-4551. [CrossRef] [PubMed]

13. Ratia, K.; Saikatendu, K.S.; Santarsiero, B.D.; Barretto, N.; Baker, S.C.; Stevens, R.C.; Mesecar, A.D. Severe acute respiratory syndrome coronavirus papain-like protease: Structure of a viral deubiquitinating enzyme. Proc. Natl. Acad. Sci. USA 2006, 103, 5717-5722. [CrossRef] [PubMed]

14. Spiegel, M.; Pichlmair, A.; Martínez-Sobrido, L.; Cros, J.; García-Sastre, A.; Haller, O.; Weber, F. Inhibition of $\beta$ interferon induction by severe acute respiratory syndrome coronavirus suggests a two-step model for activation of interferon regulatory factor 3. J. Virol. 2005, 79, 2079-2086. [CrossRef] [PubMed]

15. Frieman, M.; Ratia, K.; Johnston, R.E.; Mesecar, A.D.; Baric, R.S. Severe acute respiratory syndrome coronavirus papain-like protease ubiquitin-like domain and catalytic domain regulate antagonism of IRF3 and NF-KB signaling. J. Virol. 2009, 83, 6689-6705. [CrossRef] [PubMed] 
16. Sun, L.; Xing, Y.; Chen, X.; Zheng, Y.; Yang, Y.; Nichols, D.B.; Clementz, M.A.; Banach, B.S.; Li, K.; Baker, S.C.; et al. Coronavirus papain-like proteases negatively regulate antiviral innate immune response through disruption of STING-mediated signaling. PLOS ONE 2012, 7, e30802. [CrossRef] [PubMed]

17. Chen, X.; Yang, X.; Zheng, Y.; Yang, Y.; Xing, Y.; Chen, Z. SARS coronavirus papain-like protease inhibits the type I interferon signaling pathway through interaction with the STING-TRAF3-TBK1 complex. Protein Cell 2014, 5, 369-381. [CrossRef] [PubMed]

18. Kovalenko, A.; Chable-Bessia, C.; Cantarella, G.; Israël, A.; Wallach, D.; Courtois, G. The tumour suppressor CYLD negatively regulates NF-KB signalling by deubiquitination. Nature 2003, 424, 801-805. [CrossRef] [PubMed]

19. Liu, S.; Lv, J.; Han, L.; Ichikawa, T.; Wang, W.; Li, S.; Wang, X.L.; Tang, D.; Cui, T. A pro-inflammatory role of deubiquitinating enzyme cylindromatosis (CYLD) in vascular smooth muscle cells. Biochem. Biophys. Res. Commun. 2012, 420, 78-83. [CrossRef] [PubMed]

20. Parvatiyar, K.; Harhaj, E.W. Regulation of inflammatory and antiviral signaling by A20. Microbes Infect. 2011, 13, 209-215. [CrossRef] [PubMed]

21. Li, Y.; Chen, M.; Cao, H.; Zhu, Y.; Zheng, J.; Zhou, H. Extraordinary GU-rich single-strand RNA identified from SARS coronavirus contributes an excessive innate immune response. Microbes Infect. 2013, 15, 88-95. [CrossRef] [PubMed]

22. Cervantes-Barragan, L.; Züst, R.; Weber, F.; Spiegel, M.; Lang, K.S.; Akira, S.; Thiel, V.; Ludewig, B. Control of coronavirus infection through plasmacytoid dendritic-cell-derived type I interferon. Blood 2007, 109, 1131-1137. [CrossRef] [PubMed]

23. Devaraj, S.G.; Wang, N.; Chen, Z.; Chen, Z.; Tseng, M.; Barretto, N.; Lin, R.; Peters, C.J.; Tseng, C.T.; Baker, S.C.; et al. Regulation of IRF-3-dependent innate immunity by the papain-like protease domain of the severe acute respiratory syndrome coronavirus. J. Biol. Chem. 2007, 282, 32208-32221. [CrossRef] [PubMed]

24. Li, S.W.; Lai, C.C.; Ping, J.F.; Tsai, F.J.; Wan, L.; Lin, Y.J.; Kung, S.H.; Lin, C.W. Severe acute respiratory syndrome coronavirus papain-like protease suppressed $\alpha$ interferon-induced responses through downregulation of extracellular signal-regulated kinase 1-mediated signalling pathways. J. Gen. Virol. 2011, 92, 1127-1140. [CrossRef] [PubMed]

25. Li, S.W.; Yang, T.C.; Wan, L.; Lin, Y.J.; Tsai, F.J.; Lai, C.C.; Lin, C.W. Correlation between TGF- $\beta 1$ expression and proteomic profiling induced by severe acute respiratory syndrome coronavirus papain-like protease. Proteomics 2012, 12, 3193-3205. [CrossRef] [PubMed]

26. Clark, K.; Plater, L.; Peggie, M.; Cohen, P. Use of the pharmacological inhibitor BX795 to Study the Regulation and Physiological Roles of TBK1 and IкB Kinase $\varepsilon$ : A distinct upstream kinase mediates Ser-172 phosphorylation and activation. J. Biol. Chem. 2009, 284, 14136-14146. [CrossRef] [PubMed]

27. Yang, T.C.; Li, S.W.; Lai, C.C.; Lu, K.Z.; Chiu, M.T.; Hsieh, T.H.; Wan, L.; Lin, C.W. Proteomic analysis for Type I interferon antagonism of Japanese encephalitis virus NS5 protein. Proteomics 2013, 13, 3442-3456. [CrossRef] [PubMed]

(C) 2016 by the authors; licensee MDPI, Basel, Switzerland. This article is an open access article distributed under the terms and conditions of the Creative Commons Attribution (CC-BY) license (http://creativecommons.org/licenses/by/4.0/). 